\title{
A Design of the Traveling Recorder for Mine Locomotive
}

\author{
Sui Tao \\ Shandong University of Science and Technology \\ Qingdao China \\ sui_t@163.com \\ Huang Yong \\ Space Star Technology Co.Ltd \\ Xian China \\ huangyong7795@126.com
}

\author{
Zhou Gang \\ Yankuang Group Co. Ltd \\ Jining China \\ ykscjskf@163.com \\ Wang Jing Feng \\ Shandong University of Science and Technology \\ Qingdao China \\ 952202565@qq.com
}

\begin{abstract}
In this paper, the traveling recorder for mine locomotive is designed, which can store a section of video for further analysis and transfer the real-time image by the wireless communication to the monitoring platform. By the recorder, the personnel in the monitor room could find some hazard situation in time. The recorder can detect the image using the method of the $\mathbf{H . 2 6 4}$ codec and transfer the image by the Wi-Fi. It could be applied to monitor the running mine locomotive so as to improve the level of the management and efficiency of the mine transportation.
\end{abstract}

Key words: traveling recorder; remote image monitoring; H.264 codec, Wi-Fi

\section{PREFACE}

Underground of the coal mine, mine locomotive often use for transporting the material and tools. However, there exists many troublesome which seriously affect running safety and transport efficiency, for example, the interruption and congestion of roads, the fault and idleness of locomotive, the separation of carriage and so on. The design aims at ensuring the security of transport, benefiting running management and improving transfer efficiency.

\section{SYSTEM REVIEW}

In order to realize the remote image monitoring and management of the mine locomotive, the whole monitoring system mainly includes three parts as follows: wireless mine locomotive traveling recorder, the image transfer system, the monitoring platform. The recorder which is installed on the locomotive can not only acquire, compress and store image, but also transmit wireless WiFi. Video transmission mainly includes $\mathrm{Wi}-\mathrm{Fi}$ wireless hotpot and the fiber cable installed along the roadways. The center of monitoring platform is sited on the surface of the ground which facilitates staff to monitor running image of the mine locomotive, to locate and schedule the locomotive. The entire schematic diagram is shown in Fig.1:

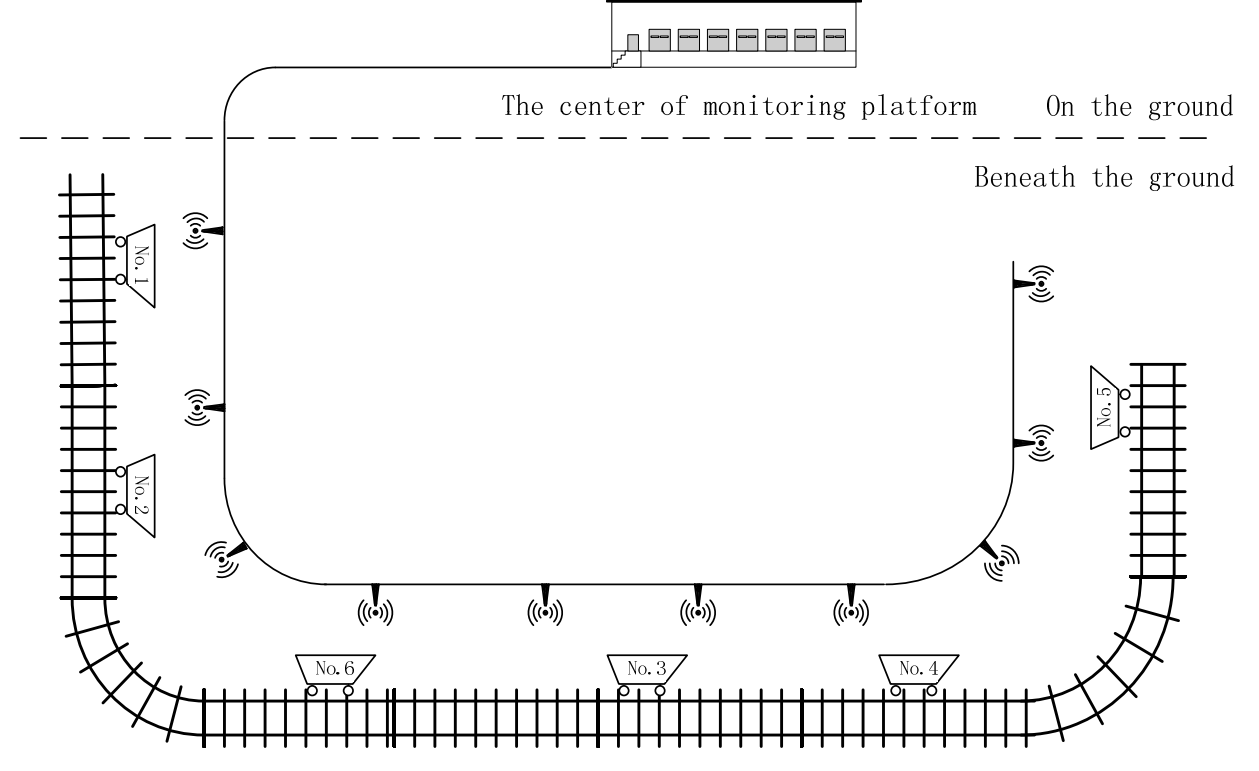

Figure 1. The entire schematic diagram of the system 


\section{THE DESIGN OF TRAVELING RECORDER}

This paper mainly introduces mine locomotive traveling recorder. It settles down the wireless transfer bandwidth limitation, ensure the image quality, and achieves real-time monitoring by using the H.264 compression standards and wireless $\mathrm{Wi}-\mathrm{Fi}$ transmission technology. The details are as follows.

\section{A. Installation of Camera and Its Monitoring Scope}

The mine roadway is too dim if we only adopt common lighting systems. The high resolution infrared cameras are used to improve more clear image. Besides, two cameras are installed on the locomotive. One observes the front roads, the other views the backward carriage and the behind roads. The two of cameras link to the record, which could expand the monitor range. The schematic diagram is shown in Fig.2:

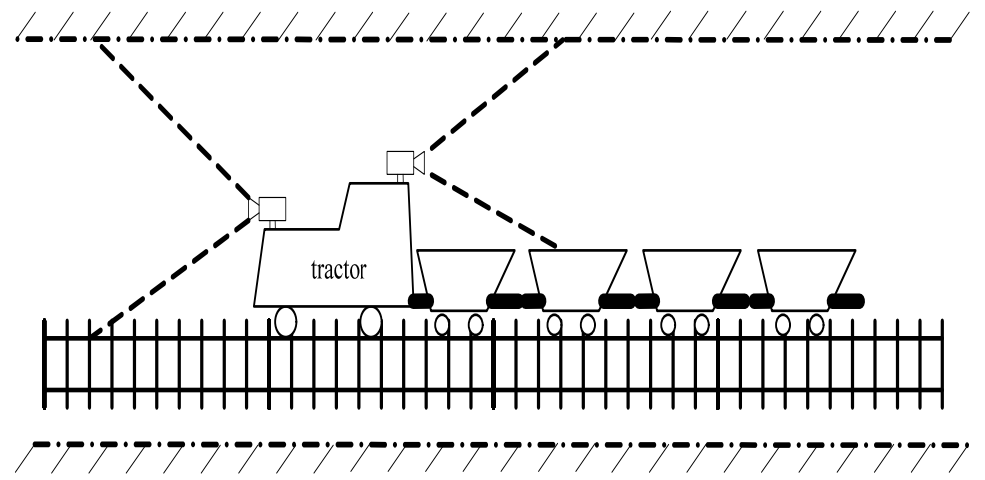

Figure 2. Installation of camera and monitoring scope

\section{B. Image Acquisition and Compression}

The quality and rate are two key factors of image compression. Firstly, the quality will not only have an impact on image decoding reconstruction clarity, but on playback of image and monitoring results of remote video. Besides, the level of compression rate will affect the image storage capacity and the wireless transmission efficiency and so on.

To realize high quality compression, the design exploits ARM Cortex-A8 core-S5P-V210processor produced by Samsung to implement acquisition and compression. The diagram is shown in Fig.3:

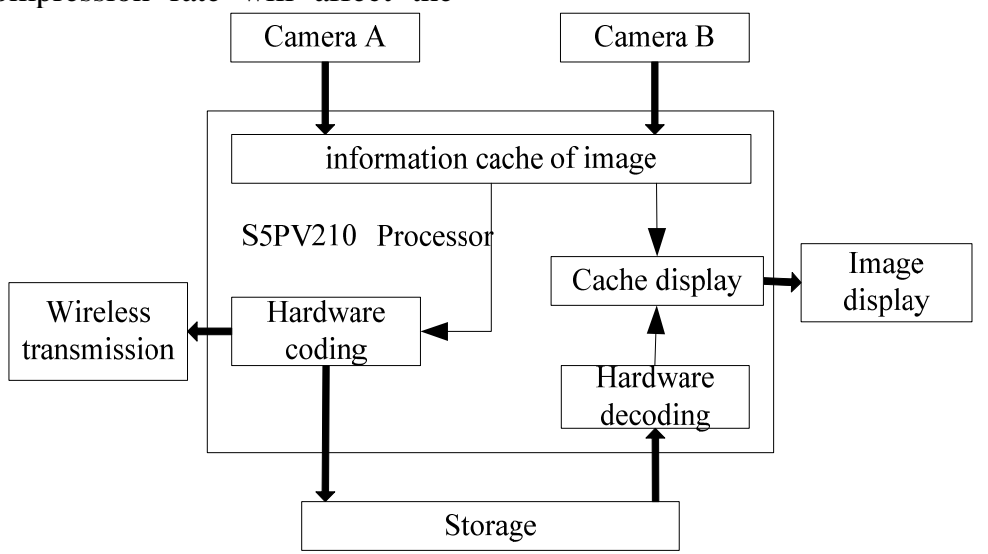

Figure 3. Image compression and storage system

\section{1) Image-reading}

In Linux operating system, we can use Video 4 Linux 2 (V4L2 for short) to operate video equipments. V4L2 defines data structure and communication mode between application program, the kernel and camera driver, interface functions supplied by V4L2 can be used to set up the camera' s frequency, frame frequency, image parameters, clipping, scaling and so on . Image acquisition is completed by the way of memory mapping based on V4L2.

\section{2) Format conversion}

Before coding, it still needs to convert format of image obtained from the camera. S5PV210 processor has two MFC coding memory formats, linear mode and
64 x 32 tiled mode are inclusive. Different formats are set up by setting the [1:0] bit of ENC_MAP_FOR_CUR .Usually, two modes are of the same effect. However, when carrying out writing operation, tiled mode require to devided $\mathrm{Y}$ and UV Component into blocks with $64 \times 32$ size, then image data is written to memory according to a certain order, data storage are more complex compared with Linear mode. Therefore, linear mode is generally selected in MFC image coding process. Commonly, the collected YUV420 data is of YV12 format, before writing data into MFC cache memory, the data should be firstly converted into NV12 format. 


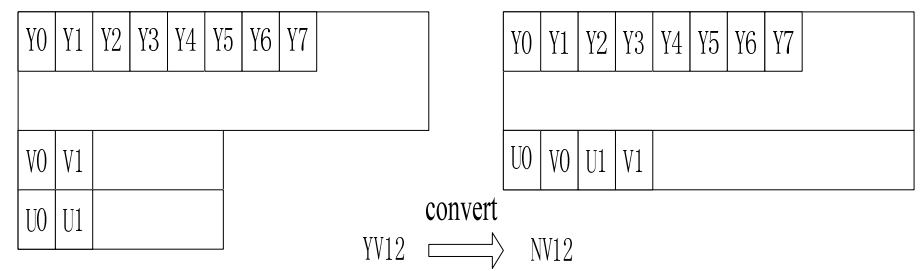

Figure 4. Format conversion diagram

\section{3) Image coding}

S5PV210 adopts ARM Cortex-A8 kernels which includes many powerful function in hardware codingdecoding aspects, and it also contains MFC so that it can supports lots Formats such as MPEG - 1/2/4, H. 263, H. 264. H. 264 has advantages over other existing standards, it has lower rate, high quality images, stronger fault tolerance, better network adaptability, as a result, excellent quality image was provided to us under the same bandwidth. With the aid of samsung's website MFC library functions, H. 264 coding comes true, the flow chart is shown in Fig.5:

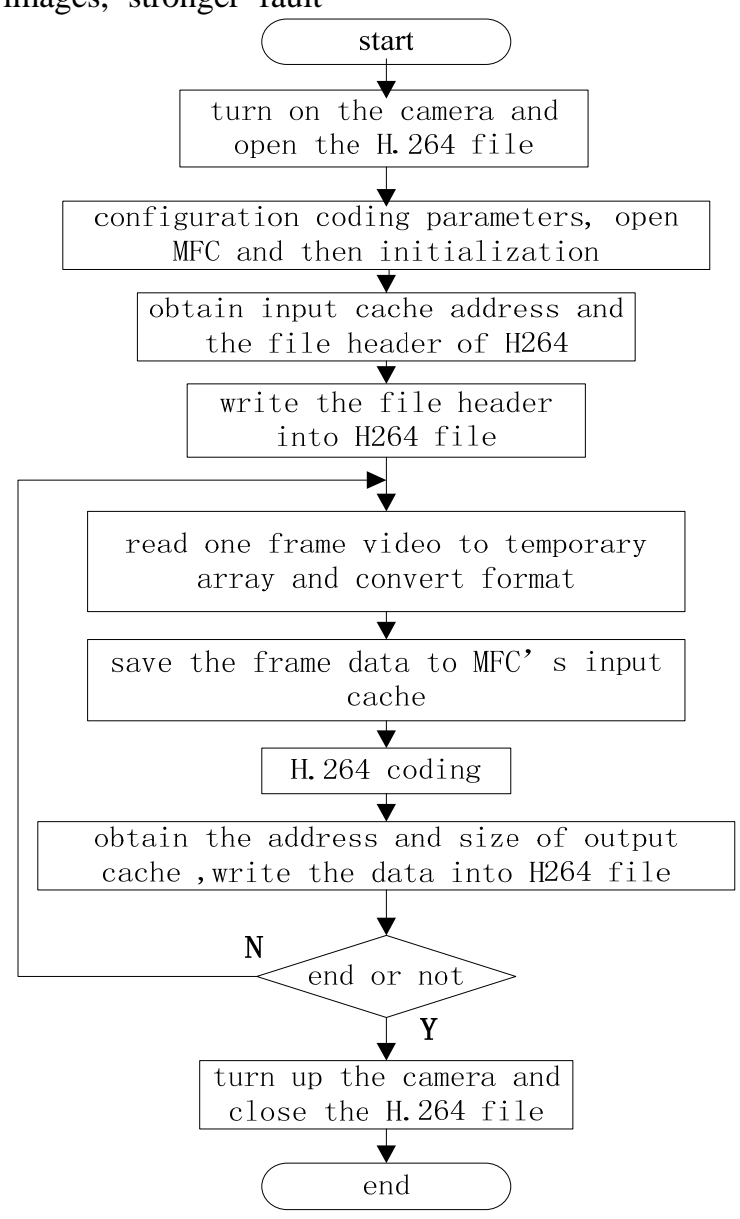

Figure 5. Hardware coding diagram of H.264

\section{4) H.26 Compressed frame}

In the process of video image compression, the compression frame of image mainly contains three types: image frame coded inside frame (I frame), predictive coding frame of image ( $\mathrm{P}$ frame), bi-directional predictive coding frame of image (B frame). On the basis of Samsung S5PV210 processor's hardware compression, we can configure compression frame format by setting
B_FRM_CTRL and I_FRM_CTRL inside the register. For example, when B_FRM_CTRL and I_FRM_CTRL are respectively set to 1and 3 , image compression frame varied in the following order: I - P - B - P - B - I. Under the circumstance of the same image environment and different pixels, the compression frame data is shown in Table 1. 
TABLE I. THE COMPRESSION FRAME DATA

\begin{tabular}{|c|c|c|c|c|c|c|}
\hline Resolution(Byte) & I Frame & P Frame & B Frame & P Frame & B Frame & I Frame \\
\hline $640 * 480$ & 35785 & 20808 & 9753 & 19756 & 9818 & 36518 \\
\hline $480 * 272$ & 12944 & 5894 & 2334 & 5893 & 1950 & 12785 \\
\hline $320 * 240$ & 6365 & 2461 & 701 & 2280 & 749 & 6324 \\
\hline
\end{tabular}

H. 264 standard has higher data compression rate, in the same image quality condition , H. 264 compression ratio is 2 times more than mpeg-2, mpeg-4 is $1.5 \sim 2$ times . From the data in table 1, in contrast, I and P frame compression ratio is not so higher as $\mathrm{B}$ because the former has to guarantee the quality of the reconstructed image. Therefore, we can give reasonable configuration based on image compression ratio and image quality to meet actual demands.

\section{Image Information Network Transmission}

Under the mine, the design uses Wi-Fi to realize fast image transmission for the convenience of mine locomotive running, it can guarantee the continuous image signal transmission through Wi-Fi hotspots automatic roaming. Finally, on the basis of Linux operating system, we construct an embedded Web server, apply standard interface of Socket network programming, adopt TCP/IP transfer protocol, and realize remote wireless video monitoring.

Finally, the ground monitoring center can monitor mine locomotives, real-time image and manage its operation by installing traveling recorder on it. Staff can observe real-time running state to ensure its safety. It is convenient for staff to schedule mine locomotive, avoid its spare and road congestions effectively, and improve transport efficiency based on locomotive localization. At the same time, the mobile operations of mine locomotive play a certain auxiliary role in monitoring safety.

\section{CONCLUSION}

Wireless mine locomotive traveling recorder is based on Linux system, it completes H. 264 image compression standard and wireless transmission by using the A8 core $\mathrm{CPU}$ to ensure the real-time and stability of remote image monitoring. System adopts modular design, it has the trait of convenient application, easily upholding so its installation and debugging don' $t$ affect the ongoing coal mining. The application of the recorder greatly benefits safety monitoring and operation management, accelerates construction proceedings of automation and information. When combined with practice, the recorder can be extended to other monitoring field also.

\section{ACKNOWLEDGMENT}

This paper is supported by the home visiting scholar program of Shandong province for the young University Key Teacher

\section{REFERENCES}

[1] LIU Fu-qiang,WANG Xin-hong, SONG Chun-lin. Digital video processing and communication [M]. Beijing: Mechanical industry publishing house, 2010.

[2] WEI Dong-shan. Embedded Linux application and development manual [M]. Beijing: People's posts and telecommunications publishing house, 2008.

[3] CAI Liyan. Based on h. 264, research and implementation of embedded network video monitoring system [D]. Nanjing: Nanjing university of posts and telecommunications, 2013.

[4] CHENG Shaolian. Based on h. 264, the research and implementation of embedded video monitoring system [D]. Wuhan: Wuhan university of science and technology, 2012.

[5] Samsung Semiconductor. S5PV210 RISC Microprocessor. Revision 1.10[EB/OL], May 2010.

[6] HUANG Xiaofeng. Application of the WiFi wireless communication system in No. 1 mine of the Pingshuo of shanxi province[J],Digital Technology and Application,2011(6):78-79.

[7] LI Ming. KT130 WiFi mine wireless communication system[J]. Industry and Mine Automation. 2010(4): 10.

[8] Wang Shuling. WIFI wireless video transmision technology in coal mine[J]. Science and Technology Innovation Herald. 2013(6): 41-42.

[9] LI Xiaoyang. WIFI technology and its application and development[J]. Information technology. 2012(2).

[10] Jonathan Corbet, Greg Kroah-Hartman, Alessandro Rubini. Linux Device Drivers[M]. O’ Reilly. February 2005. 